\title{
The Stellar Imager (SI) Mission Concept
}

Kenneth G. Carpenter ${ }^{a}$, Carolus J. Schrijver ${ }^{b}$, Richard G. Lyon ${ }^{c}$, Lee G. Mundy ${ }^{d}$, Ronald J. Allen ${ }^{e}$, J. Thomas Armstrong $f$, William C. Danchi ${ }^{g}$, Margarita Karovska ${ }^{h}$, Joe Marzouk ${ }^{i}$, Lisa M. Mazzuca ${ }^{a}$, David Mozurkewich ${ }^{f}$, Susan G. Neff ${ }^{a}$, Thomas A. Pauls $f$, Jay Rajagopal ${ }^{e}$, Gregory Solyar ${ }^{c}$, and Xiaolei Zhang $f$

${ }^{a}$ NASA's GSFC, Lab. for Astron. \& Solar Physics, Code 681, Greenbelt, MD 20771, USA

${ }^{b}$ Lockheed-Martin Advanced Technology Center, Palo Alto, CA, 94304, USA

${ }^{c}$ NASA's GSFC, Code 930 NASA-GSFC, Greenbelt, MD 20771, USA

${ }^{d}$ Astronomy Dept., Univ. of Maryland, College Park, MD 20742, USA

eSpace Telescope Science Institute, 3700 San Martin Drive, Baltimore, MD 21218, USA

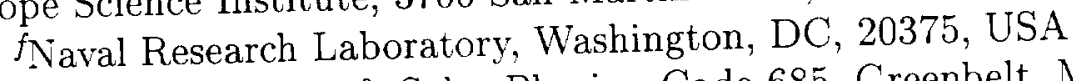

${ }^{9}$ NASA's GSFC, Lab. for Astron. \& Solar Physics, Code 685, Greenbelt, MD 20771, USA

${ }^{h}$ Harvard-Smithsonian CfA, 60 Garden Street, Cambridge, MA 02138, USA

iSigma Space, 9801 Greenbelt Rd., Suite 105, Lanham, MD 20771, USA

\begin{abstract}
The Stellar Imager (SI) is envisioned as a space-based, UV-optical interferometer composed of 10 or more onemeter class elements distributed with a maximum baseline of $0.5 \mathrm{~km}$. It is designed to image stars and binaries with sufficient resolution to enable long-term studies of stellar magnetic activity patterns, for comparison with those on the sun. It will also support asteroseismology (acoustic imaging) to probe stellar internal structure, differential rotation, and large-scale circulations. SI will enable us to understand the various effects of the magnetic fields of stars, the dynamos that generate these fields, and the internal structure and dynamics of the stars. The ultimate goal of the mission is to achieve the best-possible forecasting of solar activity as a driver of climate and space weather on time scales ranging from months up to decades, and an understanding of the impact of stellar magnetic activity on life in the Universe. In this paper we describe the scientific goals of the mission, the performance requirements needed to address these goals, the "enabling technology" development efforts being pursued, and the design concepts now under study for the full mission and a possible pathfinder mission.
\end{abstract}

Keywords: stellar activity, space-based interferometry, ultraviolet, optical, imaging

\section{INTRODUCTION}

The primary science goals of the SI mission are to study the spatial and temporal stellar magnetic activity patterns in a sample of stars covering a broad range of activity level, in order to:

- understand the underlying dynamo process(es) to enable improved forecasting of solar activity on time scales of days to centuries

- understand the impact of stellar magnetic activity on astrobiology

- enable asteroseismology to measure internal stellar structure and rotation and their relationship to the dynamo, and

Further author information:

K.G.C.: E-mail: kgc@stargate.gsfc.nasa.gov

C.J.S.: E-mail: schryver@lmsal.com 
- complete the assessment of external solar systems by imaging the central stars of systems for which the IR-interferometry missions ( TPF, IRSI/Darwin, PI) find and image planets, and to determine the impact of the activity of those stars on the habitability of the surrounding planets

SI fits naturally within the NASA and ESA long-term Strategic "Roadmaps" by complementing defined missions. With them, it will show us entire other solar systems, from the central star to their orbiting planets.

The importance of this mission to society lies in its potential to improve Space-Weather and Earth-Climate Forecasting. We must develop and validate a dynamo model in order to understand past solar activity and enable forecasting of solar and heliospheric activity days to decades in advance. This would enable us to anticipate the impact of those changes on the Earth's biosphere and society in both the long and short terms. Longterm changes, such as Maunder minima and grand maxima, can effect the climate of the earth by lowering or raising overall global temperatures, leading, for example, to crop failures. Short-term changes include enhanced activity/flares, which have the potential to disable communication satellites, knock out power grids, increase the speed of corrosion of oil pipelines, and place astronauts at risk from particle radiation.

Magnetic activity manifests itself in solar/stellar atmospheres in magnetic regions and star spots, very hot outer atmospheres, explosive flares producing high-energy particles and radiation, stellar wind and coronal mass ejections, and through the effect of these phenomena on the climates and overall habitability of orbiting planets. The main science driver of this mission is the need to understand this stellar magnetic activity, which is key to understanding life in the Universe and Earth's habitability. The stellar magnetic field slows the rotation of the collapsing cloud and thus enables star formation, couples the evolution of star and pre-planetary disk, results in energetic radiation conducive to the formation (and destruction) of complex molecules, and governs the habitability of the biosphere through its effect on space weather and planetary climate via the star's luminosity, wind, magnetic fields, and radiation. However, there is currently no comprehensive model of solar/stellar magnetic activity. This mission gathers the data needed to develop and validate such a

\section{SCIENCE, PERFORMANCE, AND DESIGN REQUIREMENTS}

There are two broad science requirements for the mission, both of which require a population study of cool stars representing a broad range of magnetic activity: imaging to detect and monitor the evolution of active regions and asteroseismology (acoustic imaging) to obtain information on the sub-surface layers. To understand the dynamo, we need to know how magnetic fields are generated and behave in different circumstances - the sun is only one example and provides insufficient constraints on theories of dynamos, turbulence, structure, and internal mixing. We must observe other stars to establish how mass, rotation, brightness and age affect the patterns of activity and determine: What determines cycle strength and duration? Can multiple cycles exist at the surface? How do polar spots form? How common is solar-like activity? What are extremely (in)active stars like? What are Maunder-minimum states like? Although its clearest manifestations are visible on the stellar surface, a full understanding of the dynamo requires a knowledge of the underlying layers: Where is the seat of the dynamo? What determines differential rotation and meridional circulation, and what role do they play in the dynamo? What is the impact of magnetic deceleration on internal rotation and stellar evolution? How are stellar interiors modified in extremely active stars?

The following primary performance goals have been established to address the science goals outlined above: (1) image a substantial sample of nearby dwarf and giant stars representing a broad range in magnetic activity, obtaining a resolution of order 1000 total pixels $(33 \times 33)$, equivalent to $\sim 50,000 \mathrm{~km}$ resolution on a Sun-like star at $4 \mathrm{pc}$., (2) study a sample in detail, revisiting over many years, and measure sizes, lifetimes, and emergence patterns of stellar active regions, surface differential rotation, field dispersal by convective motions and meridional circulation, and directly image the entire convection spectrum on giant stars and the supergranulation on, e.g., the solar counterpart Alpha Centauri and (3) enable asteroseismology, using low to intermediate degree non-radial modes to measure internal stellar structure and rotation.

The design requirements for imaging stellar surface activity include the acquisition of UV images to ensure visibility of surface manifestations of the dynamo. Dark starspots in the visible-light photosphere are 


\section{Sample Targets}

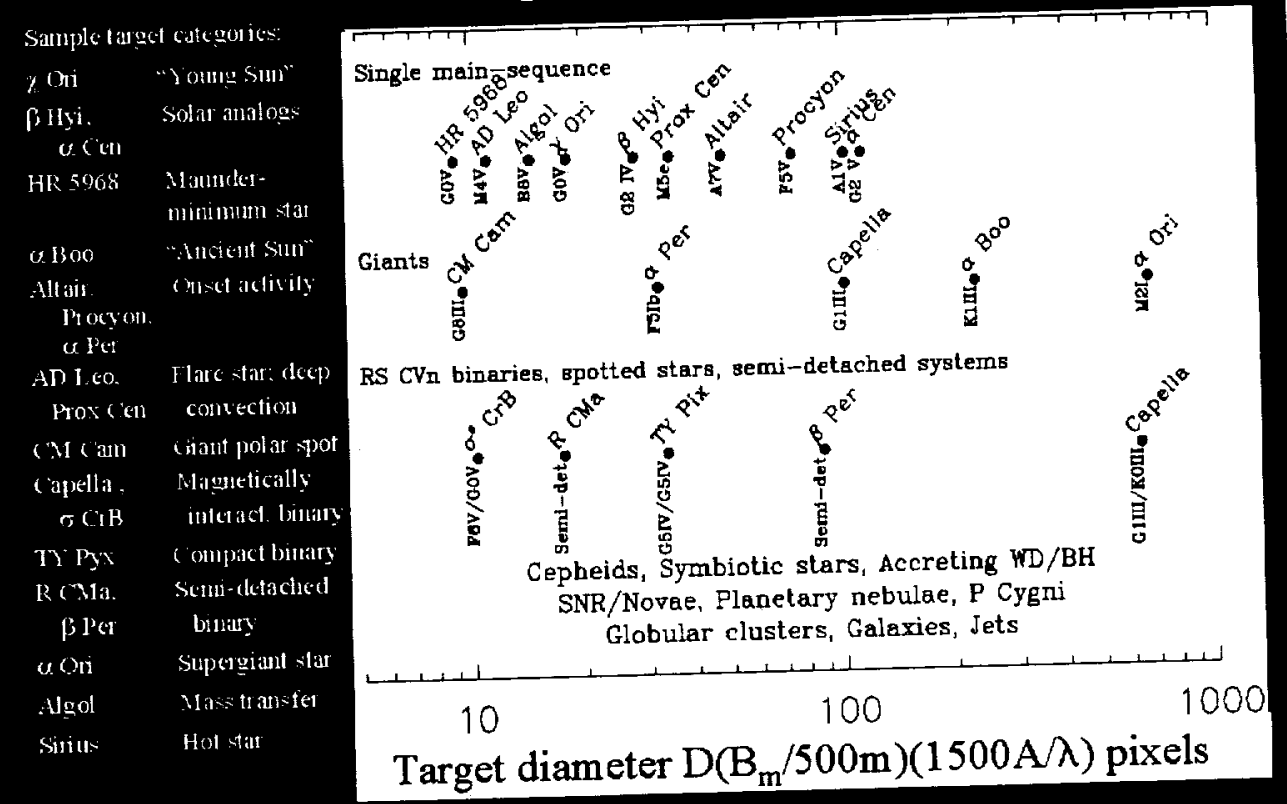

Figure 1. Estimated number of pixels across the diameter of various stars assuming a baseline of $500 \mathrm{~m}$ and a wavelength of $1500 \AA$

small in most stars and have low contrast with the surrounding bright stellar surface. High-contrast bright spots are seen in UV (chromospheric, transition-layer) emission (e.g., Mg II h\&k $2800 \AA$, C IV $1550 \AA$ ) from plages above surface wherever it is penetrated by strong magnetic fields, making them the ideal activity diagnostics. Modest integration times ( $\sim$ hours for dwarf stars to days for giant stars) are required to avoid smearing of images due to rotation, proper motions, and activity evolution.

Design requirements for imaging stellar interiors by seismology include short integration times (minutes for $\mathrm{dwarf}$ stars to hours for giant stars) and thus broadband optical wavelengths to get sufficiently high fluxes, low-resolution imaging to measure non-radial resonant waves $(\sim 30-100$ total resolution elements over the stellar surface). Flexible interferometer configurations are required for both surface and sub-surface image synthesis.

Figure 1 illustrates the range of targets one might observe with $S I$ and the apparent number of pixels that would be seen across the disk with a $500 \mathrm{~m}$ baseline at $1500 \AA$. The $S I$ array baselines could be expanded or contracted to obtain the desired number of pixels.

\section{STRAWMAN MISSION CONCEPT}

The current leading architecture concept for Stellar Imager (SI) is that of a $0.5 \mathrm{~km}$ diameter, space-based, UV-optical Fizeau Interferometer composed of a reconfigurable array of 10 - 30 one-meter-class (spherical or flat) array elements on small satellites ("mirrorsats"). Those elements direct light to an image-plane beam combination facility in a hub at the prime focus, as shown in Figure 2.

This design would provide: an angular resolution of 60 and 120 micro-arcsec at $1550 \AA$ and $2800 \AA, \sim 1000$ pixels of resolution over the surface of nearby dwarf stars, observations in $\sim 10 \AA$ UV pass-bands around, e.g., C IV $(100,000 \mathrm{~K})$ and $\mathrm{Mg}$ II h\&k $(10,000 \mathrm{~K})$, and broadband observations in the near-UV or optical continuum 


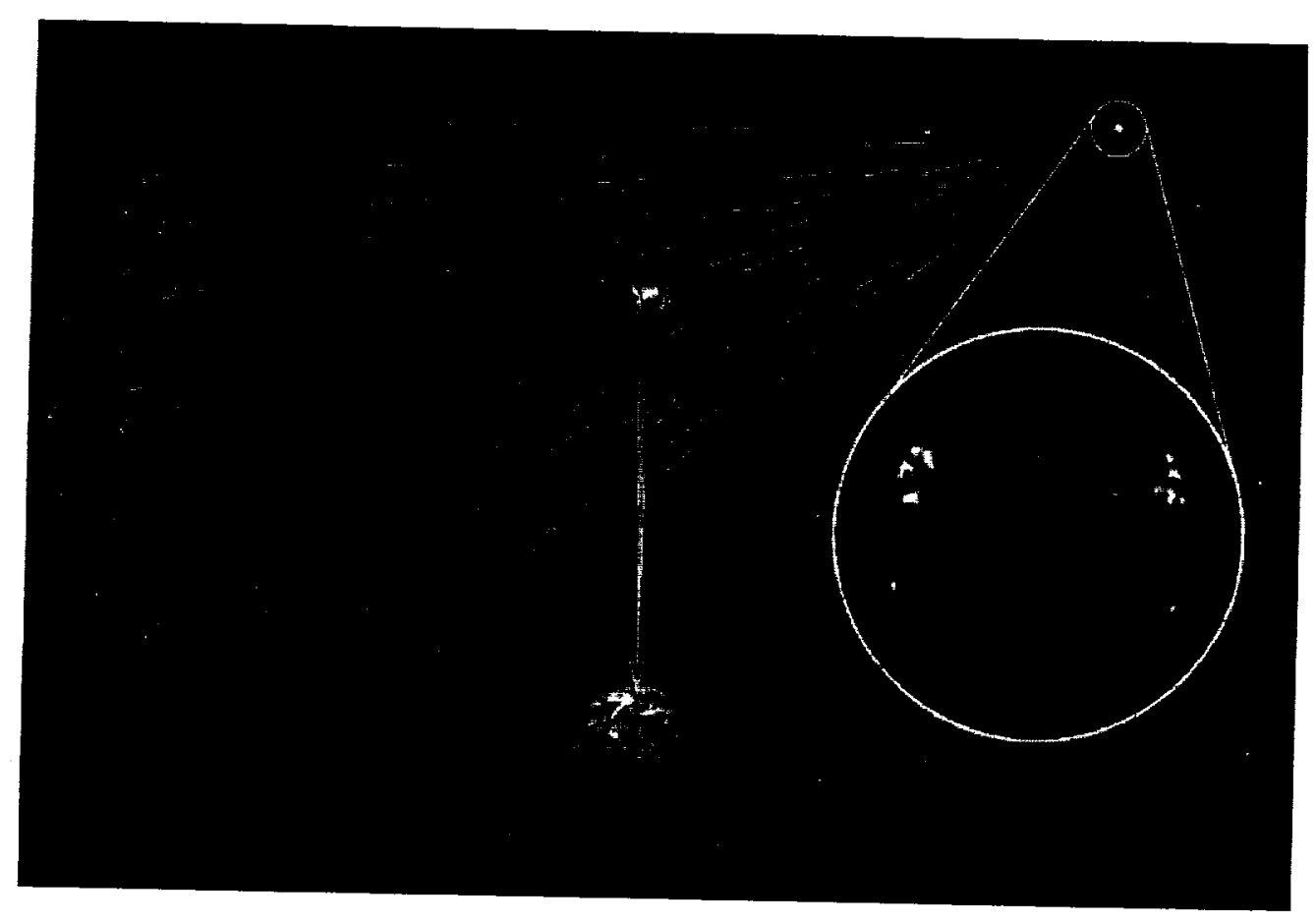

Figure 2. An artist's concept of a 21-element Fizeau Interferometer design option for SI.

(formed at 3,000-10,000 K). It is designed as a long-term mission with a requirement of a 10 year lifetime and a goal of 20 years, to allow the study of significant portions of stellar magnetic activity cycles. Individual telescopes and the central hub are designed to be refurbished or replaced as needed to support this long mission
lifetime.

$S I$ would be located in a Lissajous orbit around the sun-earth L2 point. It cannot be in low-earth orbit because the strong gravity gradient there would not permit precise formation flying (in addition to potential scattered light difficulties). An earth-trailing orbit is not desirable since replacement of failed array elements and addition of improved (larger) array elements would not be possible. L2 has both a small and very well characterized gravity gradient to permit precise formation flying and should be accessible in the 2015-2020 time frame for servicing and upgrade by robotic and/or manned missions.

A Fizeau is preferred over a Michelson design because it simplifies the beam-combination station tremendously and thus substantially lowers the cost of using many array elements. The use of many array elements enables quick acquisition of data to support imaging of transient stellar surface features (intrinsic variations, plus rotational and proper-motion-induced blurring) and high-time resolution asteroseismology, and it minimizes the number of re-configurations of the array needed to obtain the required number of baselines to ensure the desired image quality (number of baselines $\approx$ number of pixels). Other benefits include low consumption of propellant enabling the desired long-duration mission, minimizing overhead time for reconfigurations, maximizing observing efficiency and the ability to image time-dependent phenomena, and minimizing the number of reflections in the system, which is critical to maintaining UV sensitivity.

\subsection{Place in NASA/ESA Strategic Roadmaps}

$S I$ is on the strategic path of NASA Origins and ESA interferometry missions. It is a stepping stone towards crucial technology: $S I$ is comparable in complexity to the Terrestrial Planet Finder (TPF) and Darwin nullingIR-interferometers, and it may serve as a useful pathfinder for the Planet Imager (PI). SI addresses science goals of 3 NASA/Office of Space Science Thernes: understand why the sun varies (Sun Earth Connection), understand the origin of stars, planetary systems, and life (Origins), understand the structure and evolution of 


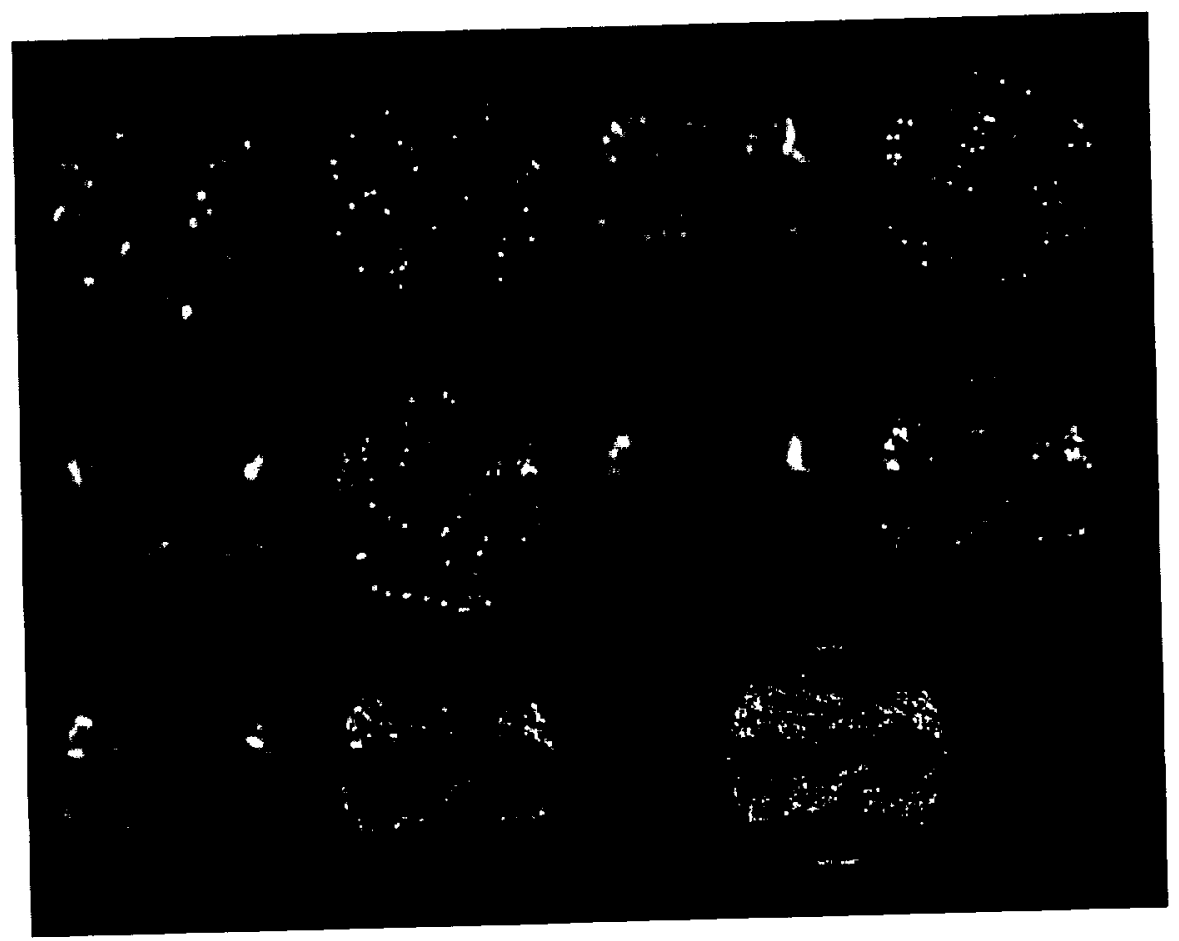

Figure 3. Simulated interferometric (CIV $(1550 \AA)$ ) images of a sun-like star at 4 pc, viewed equator-on, based on the model solar image at bottom right. See text (section 3.2 ) for details.

stars (Structure and Evolution of the Universe). It is complementary to the planetary imaging interferometers. $T P F, I R S I / D a r w i n$, and $P I$ null the stellar light to find and image planets, while $S I$ images the central star to study the effects of that star on the habitability of those planets. TPF, SI, IRSI/Darwin, and PI together provide complete views of other solar systems.

\subsection{Simulated Stellar Images}

The images that could be obtained with the strawman mission design are illustrated in Figure 3 for various numbers of elements and re-configuration strategies. These simulations were computed with SISIM (developed by R.Allen and J.Rajagopal at STScI) assuming 250 (first and third columns) and 500 (second and fourth columns) meter maximum baseline arrays. The first two rows assume Y-shaped configurations with 6 and 12 elements, respectively. The last two columns of those rows assume that the array is rotated 24 times (15 degree motions) to acquire sufficient Fourier UV-plane sampling. The 1st two images in the last row assume 30 elements arranged in a low-redundancy Golomb rectangle $\left(^{1}\right)$. The first two columns in all cases show snapshots taken without rotating the arrays. The image in the lower right is the input image.

This figure shows that 30 static elements appear to be sufficient to adequately synthesize this particular stellar image. The 435 baselines provided by the static 30 -element array works well because only about half of the 1000 pixels in the image are truly filled. If all the image pixels were filled (or a large number of the remaining pixels), then a second configuration of the array (e.g., a 90 degree rotation) would be necessary for sufficient sampling. Alternatively, fewer elements can be used with a larger number of rotations ( 6 elements with 24 rotations or 12 elements with 6 rotations).

Figure 4 compares the resolution of the 30-element design for baselines of 100,250 , and 500 meters for the same stellar model seen both equator-on and from 40 degrees north latitude. The activity belts and the larger groupings of active regions are visible at the shortest baseline, smaller groupings of plages are resolved at 250 meters, and the full 500 meter baseline is required to clearly resolve the individual active regions. 


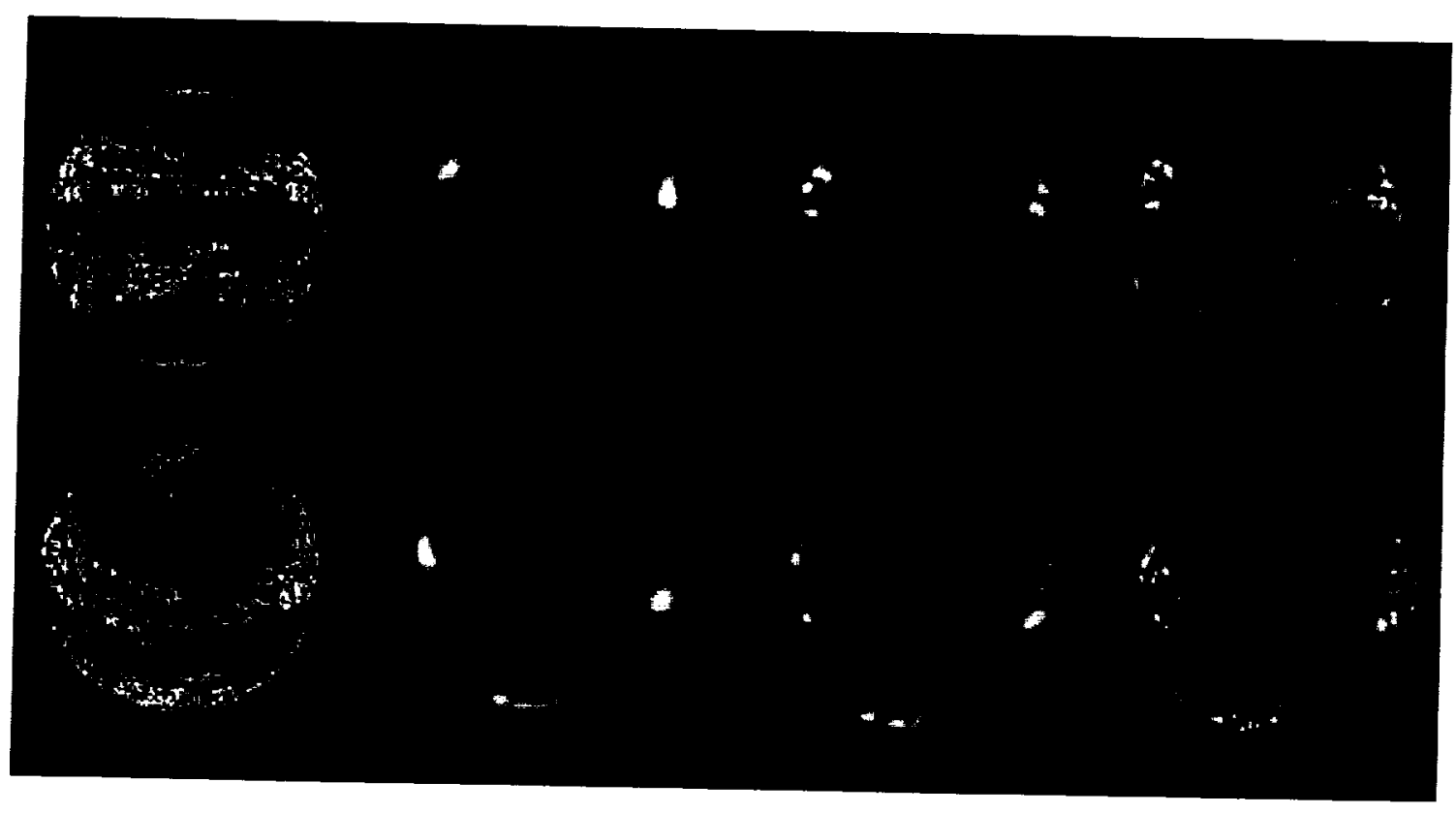

Figure 4. Simulated interferometric (CIV (1550 $\AA)$ ) images of a sun-like star at 4 pc, viewed equator-on and from 40 degrees north latitude, based on the model solar images in the first column. The last three columns show simulations for baselines of 100,250 , and 500 meters, respectively, assuming the same architecture as for the previous figure.

\subsection{Enabling Technologies}

Stellar Imager will rely on a number of critical technologies, including: precision formation flying, lightweight mirror technology, coarse ranging and array alignment, on-board autonomous computing and control systems, and closed-loop optical control to maintain array alignment based on the science data.

Study of these technologies is ongoing at NASA/GSFC, JPL, various universities, and in industry and significant leveraging and cross-fertilization will occur across projects, e.g. with NGST and TPF. A series of testbeds are in operation or are under development at GSFC, including the: Wavefront Control Testbed (WCT) to study image-based optical control methods for NGST, Phase Diverse Testbed (PDT) to study extended scene phase diversity optical control with moving array elements, Wide-Field Imaging Interferometry Testbed (WIIT) to study extending the field of Michelson imaging interferometers, and the Fizeau Interferometry Testbed (FIT) to study closed-loop control of an array of elements, as well as assess and refine technical requirements on hardware, control, and imaging algorithms. Studies of the full SI mission as well as pathfinder concepts
continue in GSFC's Integrated Design Center.

\subsection{Results of Initial GSFC Integrated Mission Design Center (IMDC) Study}

The baseline full-mission concept studied by the IMDC was for a spaced-based Fizeau interferometer, located in a Lissajous orbit around the sun-earth L2 point, with a $0.5 \mathrm{~km}$ maximum baseline and $4 \mathrm{~km}$ focal length (although this was modified in later studies to a $65 \mathrm{~km}$ focal length to allow quality imaging using simple spherical primary mirrors). The design considered included 30 mirrorsats formation flying with a beam-combining hub, where the satellites are controlled to $5 \mathrm{~nm}$ precision, rather than using optical delay lines inside the hub for fine tuning the optical path lengths. A variety of disciplines considered the implications of this general design, including power, guidance \& navigation, flight dynamics, operations, communications, quality assurance, system engineering,
etc. The highlights of this very preliminary study are summarized here.

Launch requirements are not prohibitive - three good options exist: 3 Delta III, 1 Atlas V, or 2 Delta (III/IV) launches. The preferred option is a dual launch of Delta IV 4450-14 (mirrorsats \& dispenser) + Delta III 3940-11 (hub), which allows for $30134-\mathrm{kg}$ mirrorsats + one $2600 \mathrm{~kg}$ hub. Power requirements can be handled 
by existing solar cells, but must be body-mounted to avoid unacceptable impact on precision formation-flying and station-keeping. Battery life and storage are a concern. Propellant requirements at L2 are modest: Field Emission Electric Propulsion (FEEP) should be capable in the 2015 timeframe of generating continuous, variable $\mu$-Newton thrust for required 10 year lifetime on less than $0.2 \mathrm{~kg}$ (mirrorsats) or $1 \mathrm{~kg}$ (hub) of solid fuel. The operations concept is straightforward and assumes autonomous control of array station-keeping, reconfiguration, and slewing, with ground interaction only for command uploads and anomaly resolution. The main concern of the thermal engineers is keeping mirrors isothermal.

Communications requirements are not excessive. In normal operations the mirrorsats talk to the hub and each other, and the hub talks to earth. In contingency operations: mirrorsats can be commanded directly from earth. A desired enhancement in this area would be a central communications hub at L2 for all missions flying in that locale.

Precision metrology and formation-flying are the tallest poles among numerous technical challenges. A 3level approach envisioned rough formation control via radio frequency ( $R F$ ) ranging and thrusters (to $\mathrm{m}$ 's), intermediate control (to cm's) via modulated laser ranging, and fine control (to nm's) via feedback from science data system/phase diversity analysis. The long mission lifetime requirement was the second biggest concern among the designers: the hub will have redundant components, but we need to seriously consider building a backup hub for launch-on-need or original deployment, and we would need to fly additional backup mirrorsats to put into the operating array as the original set suffers expected failures (the mirrorsats were designed as inexpensive, low-redundancy, mass-produced craft in this study).

The most important enabling technologies identified in the IMDC study as needing further study and development include: deployment/initial positioning of elements in large formations, metrology/autonomous nm-level control of many-element formations over kilometer scales, aspect control to 10 s of $\mu$ arcsecs, variable, non-condensing continuous $\mu$-Newton thrusters, light-weight UV quality spherical mirrors with $\mathrm{km}$-long radii of curvature, and larger format energy resolving detectors with finer energy resolution $(R=100)$.

\section{PATHFINDER MISSION CONCEPT}

The path to a large-baseline UV-optical interferometer in space such as SI is long and difficult. Hence, one or more pathfinder missions which take smaller technological steps and produce science results within a reasonable time-span are desirable. Existing useful precursor missions are limited: TPF may not be an interferometer at all (if the optical wavelength coronagraphic option is chosen) and, even if it is, it will be a nulling, cryogenic interferometer operating in the infrared, STARLIGHT may not fly at all (recent events have delegated it to a ground-based testbed only, at least for the moment), and SIM does not use the free-flying formations that will be needed for truly long-baseline facilities, and it will operate only at longer (optical) wavelengths and it will be used primarily as an astrometer, rather than an imager.

It would therefore be desirable to have an early mission (perhaps using booms, but free-flying if possible) with modest baselines $(\approx 20 \mathrm{~m})$, a small number of primary elements $(\approx 3-4)$, decent size mirrors $(1 \mathrm{~m})$ and the ability to perform ultraviolet beam combination and produce images in ultraviolet light. The small number of spacecraft/mirrors in this pathfinder mission would require frequent reconfigurations and therefore limit observations to targets whose variability does not preclude long integrations. However, such a mission would test most of the technologies needed for the full-mission. The addition of high-resolution spectroscopy to such a mission could increase the science return significantly at modest additional cost.

The Integrated Design Center (IDC) at GSFC has examined several possibilities in its Instrument Synthesis and Analysis Lab (ISAL) and Integrated Mission Design Center (IMDC). One of the more interesting designs is shown in Figure 5. It uses three one-meter mirrors (sections of a larger virtual parabola) on an 8.4 meter boom, a secondary mirror above them on an 18 meter mast, and a completely separate free-flying spacecraft with a single, deformable mirror, whose surface is adjustable to match the figure needed at a given location on the large virtual mirror surface, shown in Figure 6. 


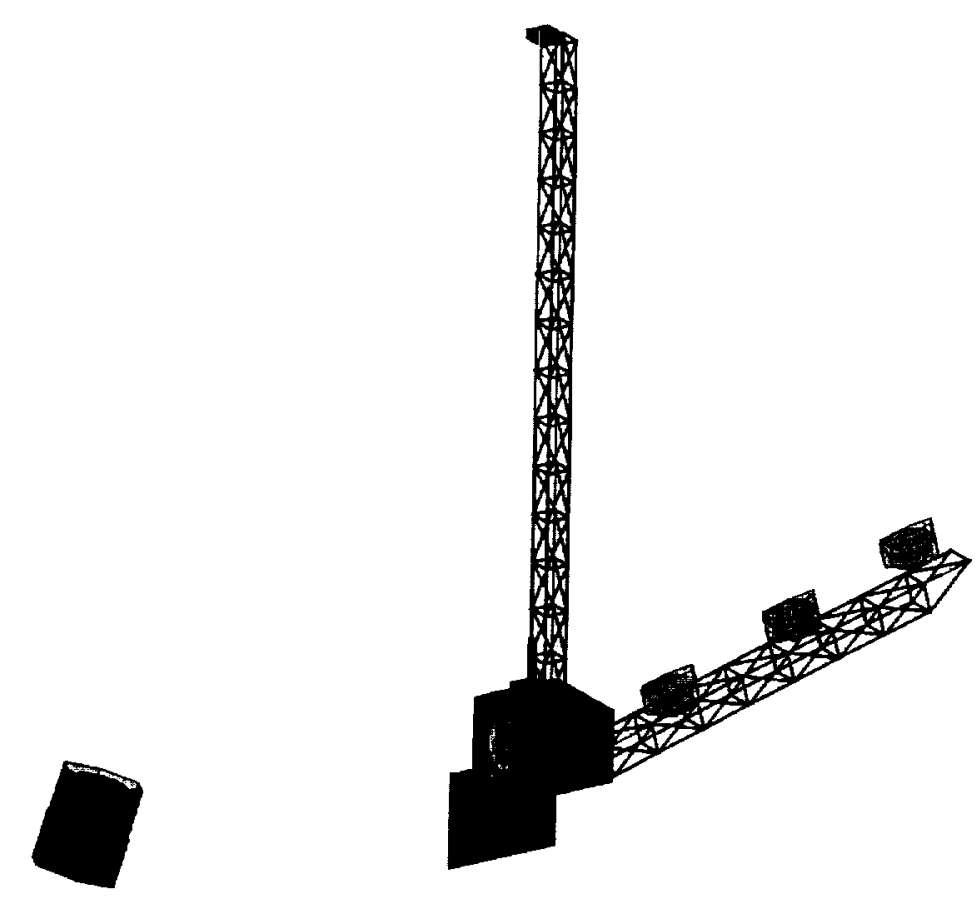

Figure 5. A concept for a UV/optical imaging interferometer pathfinder mission, utilizing 3 primary mirrors on a boom and a fourth on a free-flying spacecraft, and a secondary mirror on a mast.

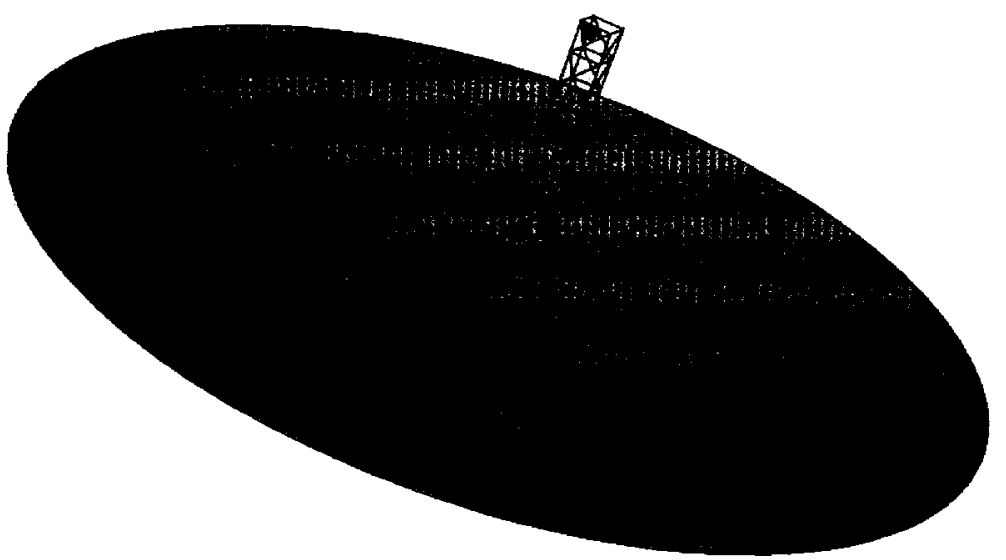

Figure 6. A view of the Pathfinder mission concept with the "virtual array" drawn in to show the surface along which the free-flyer moves and on which all the primary mirrors lie. 


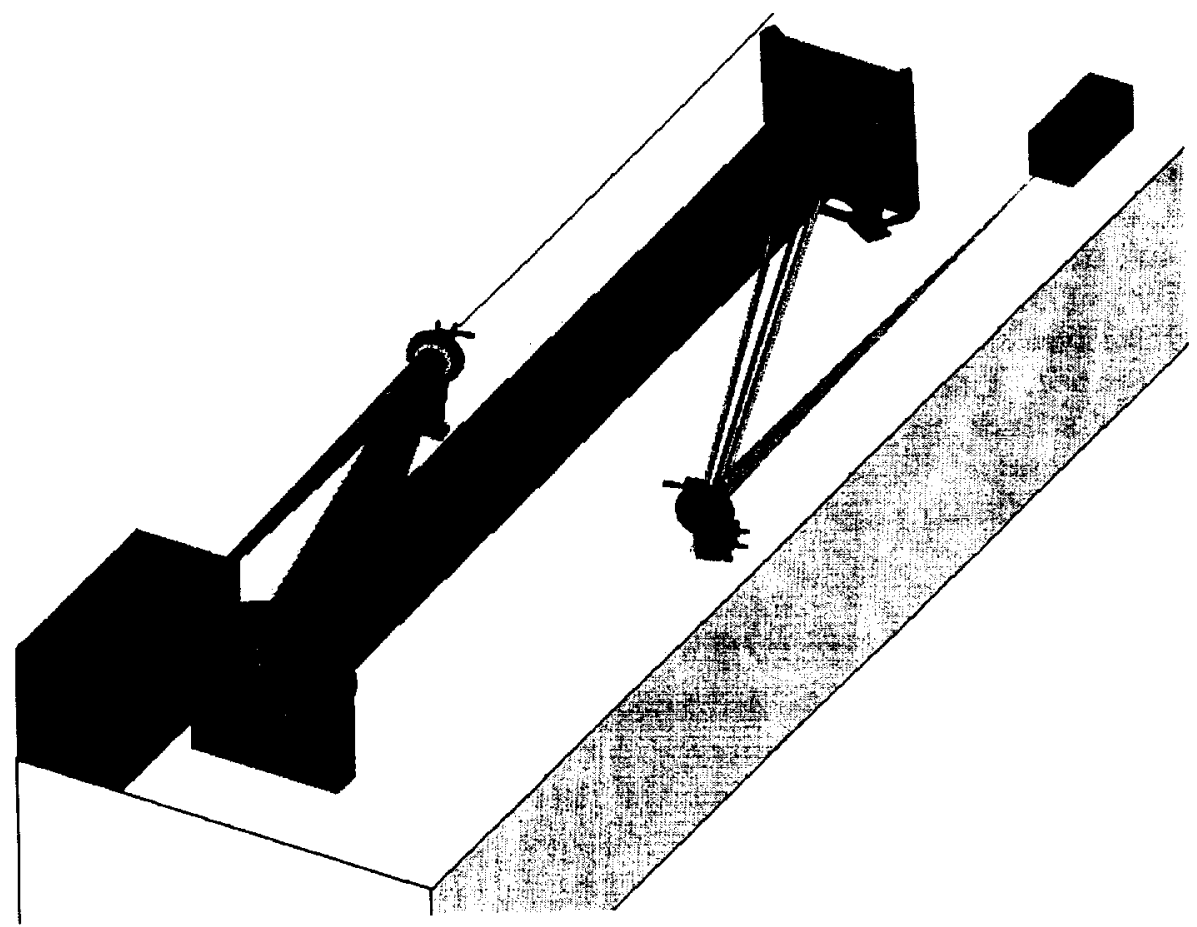

Figure 7. A schematic drawing of the Fizeau Interferometer Testbed at GSFC.

\section{GROUND-BASED TESTBEDS}

Two ground-based laboratory testbeds at GSFC for UV-Optical Fizeau Interferometers/Sparse Aperture Telescopes are being developed: the Phase Diverse Testbed (PDT) and the Fizeau Interferometry Testbed (FIT). The PDT is nearing completion now and utilizes a masked filled-aperture to simulate a system with 3 moving apertures. It enables testing of Phase Diversity algorithms which will allow the determination of the optical wavefront needed to drive control systems to maintain adequate phasing for high-resolution imaging from an array of formation-flying spacecraft. The FIT has been designed (see Figure 7) and is now under construction. It will be used to explore the principles of and requirements for the Stellar Imager mission concept and other Fizeau Interferometers/Sparse Aperture Telescope missions. It utilizes a large number of truly separate, articulated apertures (each with 5 degrees of freedom: tip, tilt, piston, and 2D translation of array elements) in a sparse distribution. It has the long-term goal of demonstrating closed-loop control of articulated mirrors and the overall system to keep beams in phase and optimize imaging. FIT also enables critical assessment of various image reconstruction algorithms (phase diversity, clean, MEM, etc.) for utility and accuracy by application to real data.

\section{SI AND GENERAL ASTROPHYSICS}

A long-baseline interferometer in space could benefit many fields of astrophysics $\left({ }^{2}\right)$. Potential targets include: Active Galactic Nuclei: the transition zone between BLR \& NLR, origin/orientation of jets; Quasi-Stellar Objects \& Black Holes: close-in structure, radiation from accretion processes; Supernovae: close-in structure; Stellar Interiors: internal structure of stars outside solar parameters; Hot Stars: hot polar winds, nonradial photospheric pulsations; Envelopes and Shells of Be-stars; Spectroscopic Binary Stars: observe companions \& orbits, determine stellar properties, perform key tests of stellar evolution; Interacting Binary Stars: resolve mass-exchange, dynamical evolution/accretion, study more efficient dynamos; Cool, Evolved Giant \& Supergiant Stars and Long-Period \& Semi-regular Variable Stars: spatio-temporal structure of extended atmospheres/winds and shocks; Forming Stars: accretion foot-points and the magnetic field structure in young star-disk systems; and Extrasolar Planet Detection: via transits. 


\section{SUMMARY/STATUS}

$S I$ is currently included in the far-horizon NASA Sun-Earth Connection Roadmap. The mission concept continues to be developed by NASA/GSFC in collaboration with LMATC, NRL/NPOI, STScI, UMD, CfA, etc. Further information on the mission can be found on the web at URL's: http://hires.gsfc.nasa.gov/ si, where a draft white paper, various science and concept presentations, and images are available for download. A Laboratory Fizeau Interferometry Testbed (FIT) is being constructed at GSFC and initial GSFC Integrated Mission Design Center (IMDC) studies of the full mission and of Pathfinder concepts have been performed. We continue with architecture and trade/feasibility studies and plan to test and demonstrate design concepts with the ground-based testbed. Finally, we plan to gather and utilize additional community input and produce a book summarizing the science and societal motivations for the mission, the technology roadmap, and the most promising architecture options. We invite you to join us in the definition and realization of this mission. Please contact K. Carpenter (kgc@stargate.gsfc.nasa.gov) or C. Schrijver (schryver@lmsal.com) with your comments and suggestions.

\section{APPENDIX A. MISSION CONCEPT DEVELOPMENT TEAM}

The Stellar Imager Mission concept is under development by NASA's Goddard Space Flight Center, in collaboration with the Naval Research Lab/Navy Prototype Optical Interferometer Group, Lockheed-Martin Advanced Technology Center, the University of Maryland, the Harvard-Smithsonian Center for Astrophysics, and the Space Telescope Science Institute, through the participation of the following individuals:

- NASA-GSFC: Ken Carpenter (Study Lead), Rick Lyon, Joe Marzouk/Sigma Space Corp., Greg Solyar/UMBC, Lisa Mazzuca, Bill Danchi, Susan Neff

- LMMS/ATC: Carolus Schrijver (Science Lead)

- NRL/NPOI: Tom Armstrong, Dave Mozurkewich, Tom Pauls, Xiaolei Zhang

- UMD: Lee Mundy

- STScI: Ron Allen, Jay Rajagopal

- CfA: Margarita Karovska

The following act as scientific and/or engineering consultants to the effort:

U Vienna: Klaus Strassmeier, U Aarhus: Jrgen Christensen-Dalsgaard, Kiepenheuer Inst: Oscar Van der Lühe, Catholic U: Fred Bruhweiler, U. Colorado: Alex Brown, Jeff Linsky, Jon Morse, BASG: Steve Kilston, CfA: Andrea Dupree, Lee Hartmann, MWO: Sallie Baliunas, SUNY: Fred Walter, Yale U: Pierre Demarque, GSFC: John Mather, Keith Gendreau, Dave Leisawitz, Juan Roman

\section{ACKNOWLEDGMENTS}

This research was supported in part by GSFC Internal Research and Development Funds, NASA Grant NAG59952 to STScI, and ROSS/SARA grants from NRA-01-01-SARA-059 for testbed development to GSFC, UMD, and NRL.

\section{REFERENCES}

1. S. Golomb and H. Taylor, Two-Dimensional Synchronization Patterns for Minimum Ambiguity, vol. IT-28, 1982.

2. P. Bely and the Space Interferometry Study Team, Kilometric Baseline Space Interferometry, vol. SCI(96)7 of ESA, European Space Agency, Paris, 1996. 